\title{
Quantum Synchronization of Two Ensembles of Atoms
}

\author{
Minghui Xu, D. A. Tieri, E. C. Fine, James K. Thompson, and M. J. Holland ${ }^{1}$ \\ ${ }^{1}$ JILA, National Institute of Standards and Technology and Department of Physics, \\ University of Colorado, Boulder, Colorado 80309-0440, USA
}

(Dated: July 24, 2013)

\begin{abstract}
We propose a system for observing the correlated phase dynamics of two mesoscopic ensembles of atoms through their collective coupling to an optical cavity. We find a dynamical quantum phase transition induced by pump noise and cavity output-coupling. The spectral properties of the superradiant light emitted from the cavity show that at a critical pump rate the system undergoes a transition from the independent behavior of two disparate oscillators to the phase-locking that is the signature of quantum synchronization.
\end{abstract}

PACS numbers: 05.45.Xt, 42.50.Lc, 37.30.+i, 64.60.Ht

Synchronization is an emergent phenomenon that describes coupled objects spontaneously phase-locking to a common frequency in spite of differences in their natural frequencies [1]. It was famously observed by Huygens, the seventeenth century clock maker, in the antiphase synchronization of two maritime pendulum clocks [2]. Dynamical synchronization is now recognized as ubiquitous behavior occurring in a broad range of physical, chemical, biological, and mechanical engineering systems $[1,3,4]$.

Theoretical treatments of this phenomenon are often based on the study of phase models [5, 6], and as such have been applied to an abundant variety of classical systems, including the collective blinking of fireflies, the beating of heart cells, and audience clapping. The concept can be readily extended to systems with an intrinsic quantum mechanical origin such as nanomechanical resonators [7, 8], optomechanical arrays [9], and Josephson junctions [10, 11]. When the number of coupled oscillators is large, it has been demonstrated that the onset of classical synchronization is analogous to a thermodynamic phase transition [12] and exhibits similar scaling behavior [13].

Recently, there has been increasing interest in exploring manifestations in the quantum realm. Small systems have been considered, e.g., one qubit [14] and two qubits [15] coupled to a quantum dissipative driven oscillator, two dissipative spins [16], two coupled cavities [17], and two micromechanical oscillators [18, 19]. Connections between quantum entanglement and synchronization have been revealed in continuous variable systems [19]. It has been shown that quantum synchronization may be achieved between two canonically conjugate variables [20]. Since the phenomenon is inherently non-equilibrium, all of these systems share the common property of competition between coherent and incoherent driving and dissipative forces.

In this paper, we propose a modern-day realization of the original Huygens experiment [2]. We consider the synchronization of two active atomic clocks coupled to a common single-mode optical cavity. It has been predicted that in the regime of steady-state superradiance [21-24] a neutral atom lattice clock could produce an ultracoherent optical field with a quality factor (ratio of frequency to linewidth) that approaches $10^{18}$. We show that two such clocks may exhibit a dynamical phase transition [26-29] from two disparate oscillators to quantum phase-locked dynamics. The onset of synchronization at a critical pump strength is signified by an abruptly increased relative phase diffusion that diverges in the thermodynamic limit. Besides being of fundamental importance in nonequilibrium quantum many-body physics, this work could have broad implications for many practical applications of ultrastable lasers and precision measurements [21].

The general setup is shown schematically in Fig. 1. Two ensembles, each containing $N$ two-level atoms with excited state $|e\rangle$ and ground state $|g\rangle$, are collectively coupled to a high-quality optical cavity. The transition frequencies of the atoms in ensembles $A$ and $B$ are detuned from the cavity resonance by $\delta / 2$ and $-\delta / 2$ respectively. This could be achieved by spatially separating the ensembles and applying an inhomogeneous magnetic field to induce a differential Zeeman shift. The atoms in both ensembles are pumped incoherently to the excited state, as could be realized by driving a transition to a third state that rapidly decays to $|e\rangle[23,24]$.

This system is described by the Hamiltonian in the rotating frame of the cavity field:

$$
\hat{H}=\frac{\hbar \delta}{2}\left(\hat{J}_{A}^{z}-\hat{J}_{B}^{z}\right)+\frac{\hbar \Omega}{2}\left(\hat{a}^{\dagger} \hat{J}_{A}^{-}+\hat{J}_{A}^{+} \hat{a}+\hat{a}^{\dagger} \hat{J}_{B}^{-}+\hat{J}_{B}^{+} \hat{a}\right),
$$

where $\Omega$ is the atom-cavity coupling, and $\hat{a}$ and $\hat{a}^{\dagger}$ are annihilation and creation operators for cavity photons. Here $\hat{J}_{A, B}^{z}=\frac{1}{2} \sum_{j=1}^{N} \hat{\sigma}_{(A, B) j}^{z}$ and $\hat{J}_{A, B}^{-}=\sum_{j=1}^{N} \hat{\sigma}_{(A, B) j}^{-}$are the collective atomic spin operators, written in terms of the Pauli operators for the two-level system $\hat{\sigma}_{(A, B) j}^{z}$ and $\hat{\sigma}_{(A, B) j}^{-}=\left(\hat{\sigma}_{(A, B) j}^{+}\right)^{\dagger}$.

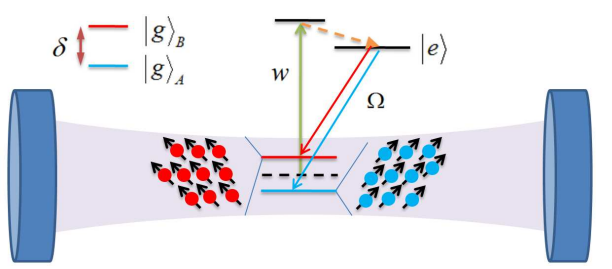

FIG. 1. (color online) Two ensembles of driven two-level atoms coupled to a single-mode cavity field. The atoms in ensemble $A$ are detuned above the cavity resonance (dashed line). Ensemble $B$ contains atoms detuned below the cavity resonance by an equivalent amount. 
In addition to the coherent atom-cavity coupling, incoherent processes are critical and include: the cavity intensity decay at rate $\kappa$, the pump rate $w$, the free-space spontaneous emission rate $\gamma$, and a background dephasing of the $|e\rangle-|g\rangle$ transition at rate $T_{2}^{-1}$. The total system is then described using a master equation for the reduced density operator $\rho$ :

$$
\begin{aligned}
\frac{d \rho}{d t}= & \frac{1}{i \hbar}[\hat{H}, \rho]+\kappa \mathcal{L}[\hat{a}] \rho+\sum_{\mathcal{T}=A, B} \sum_{j=1}^{N}\left(\gamma_{s} \mathcal{L}\left[\hat{\sigma}_{\mathcal{T} j}^{-}\right]\right. \\
& \left.+w \mathcal{L}\left[\hat{\sigma}_{\mathcal{T} j}^{+}\right]+\frac{1}{2 T_{2}} \mathcal{L}\left[\hat{\sigma}_{\mathcal{T} j}^{z}\right]\right) \rho,
\end{aligned}
$$

where $\mathcal{L}[\hat{O}] \rho=\left(2 \hat{O} \rho \hat{O}^{\dagger}-\hat{O}^{\dagger} \hat{O} \rho-\rho \hat{O}^{\dagger} \hat{O}\right) / 2$ denotes the Lindblad superoperator.

The regime of steady-state superradiance is defined by the cavity decay being much faster than all other incoherent processes [21-24]. In this regime, the cavity can be adiabatically eliminated [22], resulting in a field that is slaved to the collective atomic dipole of the two ensembles of atoms:

$$
\hat{a} \simeq-\frac{i \Omega}{\kappa+i \delta} \hat{J}_{A}^{-}-\frac{i \Omega}{\kappa-i \delta} \hat{J}_{B}^{-} .
$$

For small detuning on the scale of the cavity linewidth, $\delta \ll \kappa$, Eq. (3) reduces to $\hat{a} \simeq-i \Omega \hat{J}^{-} / \kappa$, where $\hat{J}^{-}=\hat{J}_{A}^{-}+\hat{J}_{B}^{-}$is the total collective spin-lowering operator. In this limit, the net effect of the cavity is to provide a collective decay channel for the atoms, with rate $\gamma_{c}=\Omega^{2} / \kappa$. This collective decay should be dominant over other atomic decay processes [22], i.e., $N \gamma_{c} \gg \gamma_{s}, T_{2}^{-1}$, so that the time evolution is effectively given by a superradiance master equation containing only atoms:

$$
\frac{d \rho}{d t}=\frac{\delta}{2 i \hbar}\left[J_{A}^{z}-J_{B}^{z}, \rho\right]+\gamma_{c} \mathcal{L}\left[\hat{J}^{-}\right] \rho+w \sum_{j=1}^{N}\left(\mathcal{L}\left[\hat{\sigma}_{A j}^{+}\right]+\mathcal{L}\left[\hat{\sigma}_{B j}^{+}\right]\right) \rho .
$$

With this system we naturally provide the three necessary ingredients for quantum synchronization; a controllable difference between the oscillation frequencies of two mesoscopic ensembles, a dissipative coupling generated by the emission of photons into the same cavity mode, and a driving force produced by optical pumping.

The photons emitted by the cavity provide directly measurable observables. Synchronization is evident in the properties of the photon spectra. In the case of two independent ensembles in the unsynchronized phase, each ensemble radiates photons at its own distinct transition frequency. This leads to two Lorentzian peaks that are typically well-separated. In the synchronized phase, all of the atoms radiate at a common central frequency resulting in a single peak.

To solve this problem and find the steady state, we use a semiclassical approximation that is applicable to large atom numbers. Cumulants for the expectation values of system operators $\left\{\hat{\sigma}_{(A, B) j}^{z}, \hat{\sigma}_{(A, B) j}^{ \pm}\right\}$are expanded to second order [21, 22]. All expectation values are symmetric with respect to exchange of atoms within each ensemble, i.e. $\left\langle\hat{\sigma}_{B i}^{+} \hat{\sigma}_{B j}^{-}\right\rangle=\left\langle\hat{\sigma}_{B 1}^{+} \hat{\sigma}_{B 2}^{-}\right\rangle$, for all $i \neq j$. Due to the $\mathrm{U}(1)$ symmetry, $\left\langle\hat{\sigma}_{(A, B) j}^{ \pm}\right\rangle=0$. Therefore, all nonzero observables can be expressed in terms of $\left\langle\hat{\sigma}_{(A, B) j}^{z}\right\rangle$, $\left\langle\hat{\sigma}_{(A, B) i}^{+} \hat{\sigma}_{(A, B) j}^{-}\right\rangle$, and $\left\langle\hat{\sigma}_{(A, B) i}^{z} \hat{\sigma}_{(A, B) j}^{z}\right\rangle$. Expectation values involving only one ensemble are the same for both ensembles and for these cases we omit the superfluous $A, B$ subscripts. The equations of motion can then be found from Eq. (4):

$$
\begin{aligned}
\frac{d}{d t}\left\langle\hat{\sigma}_{1}^{z}\right\rangle & =-\gamma_{c}\left(\left\langle\hat{\sigma}_{1}^{z}\right\rangle+1\right)-w\left(\left\langle\hat{\sigma}_{1}^{z}\right\rangle-1\right) \\
& -2 \gamma_{c}(N-1)\left\langle\hat{\sigma}_{1}^{+} \hat{\sigma}_{2}^{-}\right\rangle-\gamma_{c} N\left(\left\langle\hat{\sigma}_{A 1}^{+} \hat{\sigma}_{B 1}^{-}\right\rangle+\text {c.c }\right), \\
\frac{d}{d t}\left\langle\hat{\sigma}_{1}^{+} \hat{\sigma}_{2}^{-}\right\rangle= & -\left(w+\gamma_{c}\right)\left\langle\hat{\sigma}_{1}^{+} \hat{\sigma}_{2}^{-}\right\rangle+\frac{\gamma_{c}}{2}\left(\left\langle\hat{\sigma}_{1}^{z} \hat{\sigma}_{2}^{z}\right\rangle+\left\langle\hat{\sigma}_{1}^{z}\right\rangle\right) \\
& +\gamma_{c}(N-2)\left\langle\hat{\sigma}_{1}^{z}\right\rangle\left\langle\hat{\sigma}_{1}^{+} \hat{\sigma}_{2}^{-}\right\rangle \\
& +\frac{\gamma_{c}}{2} N\left\langle\hat{\sigma}_{1}^{z}\right\rangle\left(\left\langle\hat{\sigma}_{A 1}^{+} \hat{\sigma}_{B 1}^{-}\right\rangle+\text {c.c }\right), \\
\frac{d}{d t}\left\langle\hat{\sigma}_{A 1}^{+} \hat{\sigma}_{B 1}^{-}\right\rangle= & -\left(w+\gamma_{c}-i \delta\right)\left\langle\hat{\sigma}_{A 1}^{+} \hat{\sigma}_{B 1}^{-}\right\rangle+\frac{\gamma_{c}}{2}\left(\left\langle\hat{\sigma}_{A 1}^{z} \hat{\sigma}_{B 1}^{z}\right\rangle+\left\langle\hat{\sigma}_{1}^{z}\right\rangle\right) \\
& +\gamma_{c}(N-1)\left\langle\hat{\sigma}_{1}^{z}\right\rangle\left(\left\langle\hat{\sigma}_{A 1}^{+} \hat{\sigma}_{B 1}^{-}\right\rangle+\left\langle\hat{\sigma}_{1}^{+} \hat{\sigma}_{2}^{-}\right\rangle\right),
\end{aligned}
$$

describing population inversion, spin-spin coherence within each ensemble, and correlation between ensembles, respectively. In deriving Eq. (6) and (7), we have dropped third order cumulants [25]. We also factorize $\left\langle\hat{\sigma}_{(A, B) i}^{z} \hat{\sigma}_{(A, B) j}^{z}\right\rangle \approx\left\langle\hat{\sigma}_{1}^{z}\right\rangle^{2}$, which we find to be valid outside the regime of very weak pumping where a non-factorizable subradiant dark state plays an important role [22]. After making these approximations, Eq. (5) to (7) form a closed set of equations. The steady state is found by setting the time derivatives to zero and the resulting algebraic equations can be solved exactly. These solutions are the basis for the figures shown below.

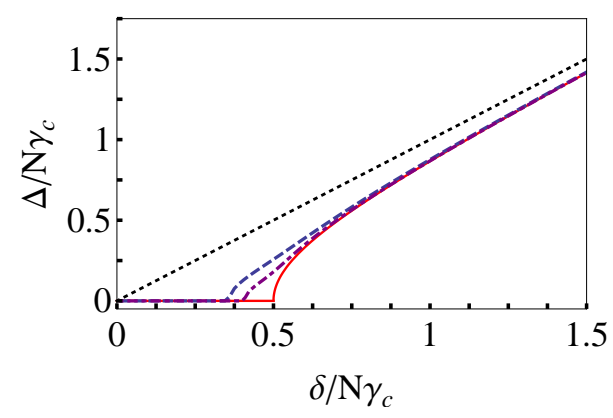

FIG. 2. (color online) Steady-state relative phase precession for two ensembles as a function of detuning at $w=N \gamma_{c} / 2$ for $N=100$ (blue dashed line), $N=500$ (purple dot dashed line) and $N=10^{6}$ (red solid line). The straight dotted line is $\delta=\Delta$.

In order to calculate the photon spectrum, we employ the quantum regression theorem [30] to obtain the two-time correlation function of the light field, $\left\langle\hat{a}^{\dagger}(\tau) \hat{a}(0)\right\rangle$, where time 0 denotes an arbitrary time-origin in steady-state. In the limit $\delta \ll \kappa$, according to Eq. (3), the phase diffusion of the atoms and light are the same, i.e. $\left\langle\hat{a}^{\dagger}(\tau) \hat{a}(0)\right\rangle \sim\left\langle\hat{J}^{+}(\tau) \hat{J}^{-}(0)\right\rangle$. We begin by deriving equations of motion for $\left\langle\hat{\sigma}_{A 1}^{+}(\tau) \hat{\sigma}_{B 1}^{-}(0)\right\rangle$ and $\left\langle\hat{\sigma}_{B 1}^{+}(\tau) \hat{\sigma}_{B 2}^{-}(0)\right\rangle$ :

$$
\frac{d}{d \tau}\left(\begin{array}{l}
\left\langle\hat{\sigma}_{A 1}^{+}(\tau) \hat{\sigma}_{B 1}^{-}(0)\right\rangle \\
\left\langle\hat{\sigma}_{B 1}^{+}(\tau) \hat{\sigma}_{B 2}^{-}(0)\right\rangle
\end{array}\right)=\frac{1}{2}\left(\begin{array}{cc}
X & Y \\
Y & X^{*}
\end{array}\right)\left(\begin{array}{l}
\left\langle\hat{\sigma}_{A 1}^{+}(\tau) \hat{\sigma}_{B 1}^{-}(0)\right\rangle \\
\left\langle\hat{\sigma}_{B 1}^{+}(\tau) \hat{\sigma}_{B 2}^{-}(0)\right\rangle
\end{array}\right),
$$


where $X=\gamma_{c}(N-1)\left\langle\hat{\sigma}_{1}^{z}(0)\right\rangle-\gamma_{c}-w+i \delta, Y=\gamma_{c} N\left\langle\hat{\sigma}_{1}^{z}(0)\right\rangle$. We have systematically factorized:

$$
\begin{aligned}
& \left\langle\hat{\sigma}_{1}^{z}(\tau) \hat{\sigma}_{A 1}^{+}(\tau) \hat{\sigma}_{B 1}^{-}(0)\right\rangle \approx\left\langle\hat{\sigma}_{1}^{z}(0)\right\rangle\left\langle\hat{\sigma}_{A 1}^{+}(\tau) \hat{\sigma}_{B 1}^{-}(0)\right\rangle, \\
& \left\langle\hat{\sigma}_{1}^{z}(\tau) \hat{\sigma}_{B 1}^{+}(\tau) \hat{\sigma}_{B 2}^{-}(0)\right\rangle \approx\left\langle\hat{\sigma}_{1}^{z}(0)\right\rangle\left\langle\hat{\sigma}_{B 1}^{+}(\tau) \hat{\sigma}_{B 2}^{-}(0)\right\rangle .
\end{aligned}
$$

Similarly, one finds that $\left\langle\hat{\sigma}_{A 1}^{+}(\tau) \hat{\sigma}_{A 2}^{-}(0)\right\rangle$ and $\left\langle\hat{\sigma}_{B 1}^{+}(\tau) \hat{\sigma}_{A 1}^{-}(0)\right\rangle$ satisfy the same equation of motion as Eq. (8). The solution of this coupled set is straightforward and shows that both $\left\langle\hat{\sigma}_{A 1}^{+}(\tau) \hat{\sigma}_{B 1}^{-}(0)\right\rangle$ and thus also $\left\langle\hat{a}^{\dagger}(\tau) \hat{a}(0)\right\rangle$ evolve in proportion to the exponential:

$$
\exp \left[-\frac{1}{2}\left(w+\gamma_{c}-(N-1) \gamma_{c}\left\langle\hat{\sigma}_{1}^{z}\right\rangle-\sqrt{\left(N \gamma_{c}\left\langle\hat{\sigma}_{1}^{z}\right\rangle\right)^{2}-\delta^{2}}\right) \tau\right]
$$

which we parametrize by $\exp [-(\Gamma+i \Delta) \tau / 2]$, where $\Gamma$ represents the decay of the first-order correlation and $\Delta$ the modulation frequency. Laplace transformation yields the photon spectrum which consists of Lorentzians of halfwidth $\Gamma / 2$ centered at frequencies $\pm \Delta / 2$.
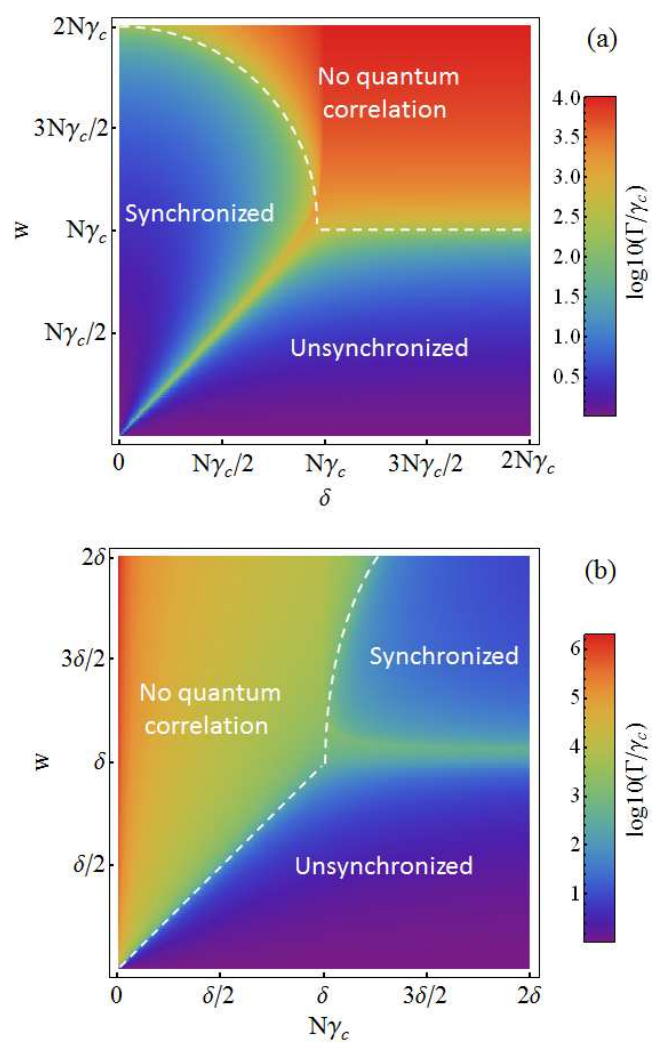

FIG. 3. (color online) (a) Nonequilibrium phase diagram of the quantum synchronization represented by $\Gamma$ (in units of $\gamma_{c}$ ) on the $w-\delta$ parameter plane, where the dissipative coupling $N \gamma_{c}\left(N=10^{4}\right)$ is fixed. An abrupt peak is observed at the boundary between the synchronized and unsynchronized phases. (b) As for (a) but on the $w-N \gamma_{c}$ parameter plane.

The importance of the two-time correlation function is that it provides direct access to the correlated phase dynamics of the two ensembles. The parameter $\Delta$ physically represents

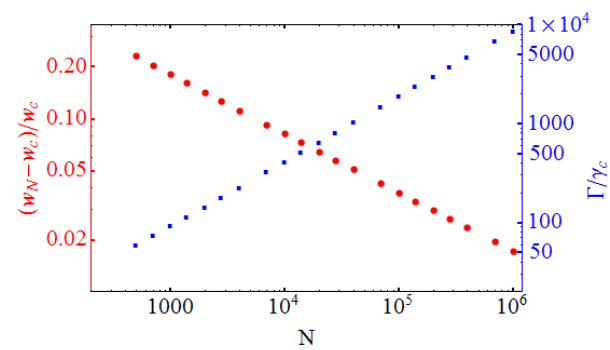

FIG. 4. (color online) Finite size scaling behavior of the quantum criticality for $\delta=N \gamma_{c} / 2$. For $N \rightarrow \infty$, the critical pump rate is $w_{c}=\delta$. The red dots show the offset between the critical pump rate $w_{N}$ for finite $N$ and $w_{c}$. The blue squares show $\Gamma$ (in units of $\gamma_{c}$ ) at $w_{N}$. Both exhibit linear scalings on the log-log plot.

the precession frequency of the phase of the collective mesoscopic dipoles with respect to one another. In Fig. 2, we show $\Delta$ as a function of $\delta$ at $w=N \gamma_{c} / 2$ for several values of $N$. For large detuning, $\Delta$ approaches $\delta$, indicating that the dipoles precess independently at their uncoupled frequency. Below a critical $\delta$, we find $\Delta$ to be zero, indicating synchronization and phase locking.

The fact that this system undergoes a synchronization transition that is fundamentally quantum mechanical and thus quite distinct from the classical synchronization previously discussed for coupled oscillators is evident in the observed properties of the linewidth $\Gamma$ of the Lorenzian peak(s), representing the relative quantum phase diffusion of the collective dipoles. This system has three independent control variables; the detuning $\delta$, the dissipative coupling $N \gamma_{c}$ and the pumping $w$, so we show $\Gamma$ on the $w-\delta$ parameter plane in Fig. 3a) and on the $w-N \gamma_{c}$ parameter plane in Fig. 3(b).

In the region of no quantum correlation, the quantum noise due to pumping destroys the coherences between spins faster than the collective coupling induced by the cavity field can reestablish them. Therefore the mesoscopic dipole is destroyed and the observed spectra are broad. In both the synchronized and unsynchronized regions, spins within each ensemble are well-correlated so that the corresponding Lorenzian peaks have ultranarrow linewidth. As is apparent in Fig. 3(a), the two ensembles cannot be synchronized when $N \gamma_{c}<\delta$ since then the coherent coupling is not sufficient to overcome the relative precession that arises from the detuning.

For strong coupling, $N \gamma_{c}>\delta$, the synchronization transition occurs as the pump rate passes a critical value. The two phases on either side of the critical region are abruptly separated. As one approaches the synchronized phase from the unsynchronized one by variation of either $\delta$ or $w$, the linewidth increases rapidly, showing amplification of the effect of quantum noise in vicinity of the critical point. After passage of the critical region, the linewidth drops rapidly, leading to rigid phase locking between the two collective dipoles.

We emphasize that the synchronization dynamics shown in Fig. 2 and 3 is a dynamical phase transition [26-29] that is reminiscent of a second-order quantum phase transition. To 
capture features of the quantum criticality, we numerically study the finite size scaling behavior. Fig. 4 shows both the critical pump rate $w_{N}$ for finite $N$ and the corresponding $\Gamma$ at $w_{N}$. The scaling laws of $\left(w_{N}-w_{c}\right) / w_{c} \simeq N^{-0.34}$ and $\Gamma / \gamma_{c} \simeq N^{0.66}$ can be identified.

In Hamiltonian systems, a quantum phase transition results from the competition between two noncommuting Hamiltonian components with different symmetries on changing their relative weight. The transition between the two distinct quantum phases can be identified from the nonanalytical behavior of an order parameter, and the scaling behavior of certain correlation functions that diverge at the critical point. By analogy, the synchronization phase transition is caused by the competition between unitary dynamics that is parametrized by $\delta$ and enters asymmetrically for the two ensembles, and drivendissipative dynamics parametrized by $\gamma_{c}$ that is symmetric. The order parameter $\Delta$ is zero in the synchronized phase and non-zero in the unsynchronized phase. The critical behavior is encapsulated by the divergence of the relative quantum phase diffusion. It should be emphasized that the treatment given here is quite different to the typical analysis since the transition is embodied by the characteristic features of the two-time correlation functions, rather than the behavior of an energy gap or correlation length.

In the thermodynamic limit, simple expressions for $\left\langle\hat{\sigma}_{1}^{z}\right\rangle$ to leading order in $1 / N$ can be obtained:

$$
\left\langle\hat{\sigma}_{1}^{z}\right\rangle=\left\{\begin{aligned}
\frac{w}{2 N \gamma_{c}}, & \text { if } \delta=0 \\
\frac{w^{2}+\delta^{2}}{2 w N \gamma_{c}}, & \text { if } 0<\delta<w, \\
\frac{w}{N \gamma_{c}}, & \text { if } \delta \geq w
\end{aligned}\right.
$$

where $w$ should be such that $\left\langle\hat{\sigma}_{1}^{z}\right\rangle<1$. A critical point at $w_{c}=$ $\delta$ can be found by substituting Eq. (11) into Eq. (10). In particular, $\Delta=\left(\delta^{2}-w^{2}\right)^{1 / 2}$ in the unsynchronized phase, which shows an analogous critical exponent to that of a second-order quantum phase transition, i.e., $\beta=1 / 2$.

In conclusion, we have presented a system that exhibits quantum synchronization as a modern analogue of the Huygens experiment but is implemented using state-of-the-art neutral atom lattice clocks of the highest precision. It will be intriguing in future work to study the many possible extensions that are inspired by these results, such as the effect of an atom number imbalance on the synchronization dynamics, and the sensitivity of the phase-locking to external perturbation.

We acknowledge stimulating discussions with J. Cooper, J. G. Restrepo, D. Meiser, K. Hazzard, and A. M. Rey. This work has been supported by the DARPA QuASAR program, the NSF, and NIST.
[1] S. H. Strogatz, Sync: The Emerging Science of Spontaneous Order (Hyperion, New York, 2003).

[2] M. Kapitaniak, K. Czolczynski, P. Perlikowski, A. Stefanski, and T. Kapitaniak, Phys. Rep. 517, 1 (2012).

[3] A. Pikovsky, M. Rosenblum, and J. Kurths, Synchronization: A Universal Concept in Nonlinear Sciences (Cambridge University Press, Cambridge, England, 2001).

[4] S. Bregni, Synchronization of Digital Telecommunications Networks (Wiley, Chichester, 2002).

[5] Y. kuramoto, Chemical Oscillations, Waves and Turbulence (Courier Dover Publications, 2003).

[6] J. A. Acebrón et al., Rev. Mod. Phys. 77, 137 (2005).

[7] M. C. Cross, A. Zumdieck, Ron Lifshitz, and J. L. Rogers, Phys. Rev. Lett. 93, 224101 (2004).

[8] C. A. Holmes, C. P. Meaney, and G. J. Milburn, Phys. Rev. E 85, 066203 (2012).

[9] G. Heinrich, M. Ludwig, J. Qian, B. Kubala, and F. Marquardt, Phys. Rev. Lett. 107, 043603 (2011).

[10] A. K. Jain, K. K. Likharev, J. E. Lukens, and J. E. Sauvageau, Phys. Rep. 109, 309 (1984).

[11] K. Wiesenfeld, P. Colet, and S. H. Strogatz, Phys. Rev. Lett. 76, 404 (1996).

[12] A. T. Winfree, J. Theor. Biol. 16, 15 (1967).

[13] O. Kogan, J. L. Rogers, M. C. Cross, and G. Refael, Phys. Rev. E 80, 036206 (2009).

[14] O. V. Zhirov and D. L. Shepelyansky, Phys. Rev. Lett. 100, 014101 (2008).

[15] O. V. Zhirov and D. L. Shepelyansky, Phys. Rev. B 80, 014519 (2009).

[16] P. P. Orth, D. Roosen, W. Hofstetter, and K. LeHur, Phys. Rev. B 82, 144423 (2010).

[17] T. E. Lee and M. C. Cross, arXiv:1209.0742 1 (2012).

[18] M. Zhang, G. S. Wiederhecker, S. Manipatruni, A. Barnard, P. McEuen, and M. Lipson, Phys. Rev. Lett. 109, 233906 (2012).

[19] A. Mari, A. Farace, N. Didier, V. Giovannetti, and R. Fazio, arXiv:1304.5925 1 (2013).

[20] A. M. Hriscu and Y. V. Nazarov, Phys. Rev. Lett. 110, 097002 (2013).

[21] D. Meiser, J. Ye, D. R. Carlson, and M. J. Holland, Phys. Rev. Lett. 102, 163601 (2009).

[22] D. Meiser and M. J. Holland, Phys. Rev. A 81, 033847 (2010); D. Meiser and M. J. Holland, Phys. Rev. A 81, 063827 (2010).

[23] J. G. Bohnet, Z. Chen, J. M. Weiner, D. Meiser, M. J. Holland, and J. K. Thompson, Nature 484, 78 (2012).

[24] J. G. Bohnet, Z. Chen, J. M. Weiner, K. C. Cox, and J. K. Thompson, Phys. Rev. Lett. 109, 253602 (2012).

[25] We have validated the closed set of Eq. (5)-Eq. (7) by comparison with exact solutions of the quantum master equation based on applying the SU(4) group theory (see Minghui Xu, D. A. Tieri, M. J. Holland, Phys. Rev. A 87, 062101 (2013)). Due to the presence of multiple ensembles it is difficult to implement exact calculations for more than about ten atoms.

[26] S. Diehl, A. Tomadin, A. Micheli, R. Fazio, and P. Zoller, Phys. Rev. Lett. 105, 015702 (2010).

[27] A. Tomadin, S. Diehl, and P. Zoller, Phys. Rev. A 83, 013611 (2011).

[28] E. M. Kessler, G. Giedke, A. Imamoglu, S. F. Yelin, M. D. Lukin, and J. I. Cirac, Phys. Rev. A 86, 012116 (2012).

[29] B. Horstmann, J. I. Cirac, and G. Giedke, Phys. Rev. A 87, 012108 (2013).

[30] M. Lax, Phys. Rev. 129, 2342 (1963); C. W. Gardiner, Quantum Noise (Springer-Verlag, Berlin, 1991). 